\title{
Subject Index to Volume 33
}

Acetylcysteine, role, reversal of chronic $2-n$ propyl-4-pentenoic acid hepatotoxicity, mice, 72

Actin, polymerization, neutrophil effect, neonate, 27

Adenine nucleotide, translocator deficiency, patient with mitochondrial myopathy, 412

Adenosine

microcirculatory responses to, newborn pig retina, 620

oxygen-induced pulmonary vasodilation and, fetal lamb, 533

transport inhibition, cardiovascular effect, after asphyxia, fetal lamb, 185

Adenosine deaminase

deficiency

biochemical abnormalities and molecular genetics, S35

enzyme replacement therapy, case reports, S42

gene therapy for, $\$ 49$

Adenosine triphosphate

oxygen-induced pulmonary vasodilation and, fetal lamb, 533

renal, thyroxin effect on recovery of, rat, 595

Age, colonic mucin composition and, infant and mature pigs, 564

Alkalosis, pulmonary vasodilation produced by, newborn lamb, 341

Allopurinol, after cerebral hypoxia-ischemia, immature rat, 405

Amino acids

excitatory, CNS injury in bacterial meningitis and, rabbit, 510

intravenous, effect on protein metabolism, preterm infants, 106

Antioxidant, altered plasma status, cystic fibrosis, 247

Antioxidant enzyme

antenatal cocaine exposure and, late gestation rat, 327

gene expression, prenatal hormone effect, fetal rat lung, 171

intratracheal instillation, hyperoxic lung injury, rat, 332

response to hyperoxia, after prenatal dexamethasone, preterm rat, 177

Aorta, in situ morphology, fetal and neonatal rat, 302

Arachidonic acid, cerebral vasodilator response to, indomethacin effect, newborn pig, 609

Asphyxia

cardiovascular function after, adenosine transport inhibition effect, fetal lamb, 185

retinal function deterioration after, ibuprofen effect, newborn pig, 336

Autonomic nervous system, regulation of hemodynamic responses by, during endotracheal suctioning, infant, 649

Betamethasone, maternal administration, effect on lung maturation, preterm rabbit, 256

Bile acid, ileal transport, bile acid feeding effects, rat, 221
Bile salt, ileal bile acid transport and, rat, 221

Biliary obstruction, extrahepatic, rotavirusassociated, newborn mice, 394

Birth, mode of delivery, effect on neonatal polymorphonuclear leukocyte function, 466

Bisulfite, parenteral administration, effects on human vascular tissue, 347

Blood, coagulation abnormalities, carbohydrate-deficient glycoprotein syndrome, 540

Blood flow

cerebral

estimation, near infrared spectroscopy and ${ }^{133}$ xenon clearance, 56

extracorporeal membrane oxygenation effect, newborn lamb, 289

phenobarbital effect, during hypotension, newborn pig, 598

epinephrine infusion effect, anesthetized piglet, 190

patterns, effects of spontaneous, rhythmic pulmonary ventilation, fetal sheep, 639

peripheral, monitoring by plethysmo time interval, neonate, 653

retinal, metabolic regulation, adenosine role, newborn pig, 620

Body fluid, compartment volumes, chronically hypoxemic lambs, 267

Brain: see also specific area

injury, allopurinol effect, immature rat, 405

repeated ischemia, neuronal consequences, sheep, 61

Breast-feeding, erythrocyte incorporation of ingested iron isotope, comparison, formula-fed infants, 573

Bronchiolitis, respiratory mechanics and occlusion time in infants with, 273

Calcium, kinetics, hyperprostaglandin E syndrome, 92

Carbohydrate-deficient glycoprotein syndrome, coagulation abnormalities, unique pattern of, 540

Carbon tetrachloride, hepatotoxicity, chlordecone effect, neonatal rat, 225

Cardiopulmonary arrest, mitochondrial disorder associated with, neonate, 433

Cardiovascular development, neural crest ablation $v s$ sham surgical effects, chick embryo, 628

Carnitine, role, reversal of chronic 2-n-propyl-4-pentenoic acid hepatotoxicity, mice, 72

Catalase activity, developmental differences, premature and full-term lamb lungs, 519

Centronuclear myopathy, X-linked, prenatal diagnosis, 201

Chlordecone, carbon tetrachloride hepatoxicity and, neonatal rat, 225

Circulation: see Blood flow

Cocaine, accelerated surfactant maturation produced by, late gestation rat, 327

Collagen, circulating metabolites, insulin-dependent diabetics with joint and skin manifestations, 501
Colon, lactose uptake, newborn piglet, 568

Commentary, Gene therapy: possibilities and promise, 321

Coronary heart disease, risk factors, plasma fibrinogen in biracial community, 323

Cow milk, effect, rotavirus-associated intestinal dysfunction, suckling rat, 548

Cystic fibrosis, altered antioxidant status, reduced free-radical-trapping capacity and, 247

Cytochrome oxidase, cerebral, indomethacin effect, preterm infant, 603

Deoxyribonucleic acid, linkage analysis, prenatal diagnosis of $\mathrm{X}$-linked centronuclear myopathy by, 201

Dermatophagoides pteronyssinus, group II antigen, epitope analysis, 209

Dexamethasone

prenatal, antioxidant enzyme response and, preterm rat, 177

regulation of ductus arteriosus smooth muscle cell migration by, fetal lamb, 352

short-term therapy, effect on zinc metabolism, piglet, 118

Diabetes mellitus, insulin-dependent, joint and skin manifestations, 501

Diaphragm, function, group B streptococcal sepsis effect, piglet, 10

Diarrhea

antigen absorption during, diet role, suckling rat, 548

Giardia lamblia-associated, cellular immune response during, 15

Diet

colonic mucin composition and, infant and mature pigs, 564

antigen absorption during diarrhea and, suckling rat, 548

Dihydropteridine reductase, deficiency, catalytic activity of tetrahydrobiopterin in, 125

2,3-Diphosphoglycerate, regulation, genetic and environmental effects, preadolescent children, 645

DNA: see Deoxyribonucleic acid

Docosahexaenoic acid, visual response related to, healthy term infants, 425

Dopamine, receptor blockade and stimulation, systemic and pulmonary vascular effects, lamb, 181

Ductus arteriosus

antenatal indomethacin effect, fetus and newborn, 615

smooth muscle cell migration, regulation of, fetal lamb, 352

Endotracheal suctioning, hemodynamic changes during, autonomic nervous system role, infant, 649

Energy, metabolism, neonate receiving TPN, 112

Enterobacteria, heat-stable enterotoxin plasmid transfer among, 205

Enterocytes, cell surface phenotypes, small intestinal mucosa, Kawasaki disease, 557

Enterotoxin, heat-stable, interspecific plas- 


\section{SUBJECT INDEX TO VOLUME 33}

mid transfer and modification of, 205

Epidermal growth factor, expression, fetal gastrointestinal tract, 481

Epinephrine, infusion, circulatory effects, piglet, 190

E1 pyruvate dehydrogenase, deficiency, child with motor neuropathy, 284

Erythrocyte

incorporation of ingested iron isotope by, breast-fed and formula-fed infants, 573

membrane fluidity, changes, psoriatic children, 506

Fatty acid

carbon chain length, mucosal injury and, immature piglet, 543

degree of saturation, mucosal injury and, immature piglet, 543

dietary supply, evaluation, formula-fed infants, 425

Fetus

antibody production, determination of anti-idiotypes, 475

antioxidant enzyme gene expression, prenatal hormone effect, rat lung, 171

barrier ontogenesis, intrauterine growthretarded rat, 418

brain lipid synthesis from lactate, rat, 66

chondrocytes, regulation of clonal growth by transforming growth factor $-\beta-1$, 390

chronic hypoglycemia and hypoinsulinemia, cytosolic phosphoenolpyruvate carboxykinase induction by, ovine liver, 493

ductus arteriosus, regulation of smooth muscle cell migration, lamb, 352

gastrointestinal tract, epidermal growth factor and transforming growth factor$\alpha$ expression, 481

hemodynamic effects of antenatal indomethacin, randomized study, 615

late-gestation growth, pituitary regulation of, rat, 144

lung

caine effect on antioxidant enzyme development, late gestation rat, 327

maturation, after maternal betamethasone, preterm rabbit, 256

oxygen-induced vasodilation, adenosine triphosphate and adenosine role, lamb, 533

retinoic acid receptors, gestational age and retinol deficiency effects, 251

repeated ischemia, neuronal consequences, sheep brain, 61

severe asphyxia, adenosine transport inhibition effect after, lamb, 185

spontaneous breathing, lung distension and, hemodynamic effect, sheep, 639

transplacental transport of serine and leucine, comparison, sheep, 590

Fibrinogen, plasma levels, correlates of, biracial community, 323

Flavin adenine nucleotide, mitochondrial levels, defective maintenance of, 129

Gastroenteritis, antigen absorption during, diet role, suckling rat, 548

Gastrointestinal tract

immunohistochemical studies, Kawasaki disease, 557

transforming growth factor- $\alpha$ and epidermal growth factor expression in, human fetus, 481

Gene transfer, hepatic, clinical protocol for, 313,321 (commentary)
Gestational age, role, regulation of retinoic acid receptors, fetal rat lung, 251

$\mathrm{GH}$ : see Growth hormone

Giardia lamblia, gastrointestinal infection, cellular immune response during, 15

Glucocorticoids

regulation of $\mathrm{Na}^{+}, \mathrm{K}^{+}$-ATPase mRNA by, developing rat lung and kidney, 5

role, regulation of $\mathrm{Na}^{+}, \mathrm{K}^{+}$-ATPase mRNA abundance, infant rat heart, 1

Glucoregulatory hormone, levels, ventilated preterm infants, 583

Glucose

deuterated, glucose production rate determined by, immature neonates, 97

oxidation, preterm infants receiving medium-chain triglycerides, 101

production and oxidation, ventilated preterm infants, 583

production rate, extremely immature neonates, 97

Glutamine, cerebral concentration, ornithine carbamoyl transferase deficiency, 77

Glyburide, correction of hyprinsulinemia by, nondiabetic patients with thalassemia major, 497

Glycine, uteroplacental production from serine, sheep, 590

Glycogen, postnatal depletion, cardiopulmonary arrest and, neonate, 433

Gonadotropin, elevated levels, early pubertal boys with testicular defects, 514

Growth and development

after acute lymphoblastic leukemia treatment, cranial irradiation effect, 577

betamethasone and lung maturation, preterm rabbit, 256

colonic mucin composition, infant and mature pigs, 564

constitutional delay, growth hormone secretion in prepubertal males with, 278

development of human hypothalamic vasopressin, 152

different regulation of clonal growth, human fetal chondrocytes, 390

environmental hypoxia effect on maximum coronary flow rates, isolated rabbit hearts, 527

fetal, isolated GH deficiency, rat, 144

fetal and neonatal aorta, in situ morphology, rat, 302

hypoxia-hyperoxia effects, preterm and full-term lamb lung, 519

ileal bile acid transport system, bile salt effects, rat, 221

multinucleated giant cell formation, umbilical cord and adult mononuclear phagocytes, 19

Growth hormone, altered secretory dynamics, short prepubertal males, 278

Growth retardation, intrauterine, barrier ontogenesis in, fetal rat, 418

Haemophilus influenzae, type b capsular polysaccharide, response, molecular basis of antibody gene diversity and, 307

Haemophilus influenzae type $\mathrm{B}$, pial vessel autoregulation impaired by, rat, 48

Heart: see also specific area

glucocorticoid role, regulation of $\mathrm{Na}, \mathrm{K}^{+}$ATPase mRNA abundance, 1

maximum coronary flow rate, growth and maturation effect, hypoxic environment, rabbit, 527

myocardial perfusion and performance, after indomethacin administration, newborn lamb, 295

Heme oxygenase inhibitor, metalloprophyrin screening and characterization, neonatal jaundice, 195

Hemodynamics

lung distension and spontaneous fetal breathing effects, sheep, 639

resting, dopaminergic influence, lamb, 181

Hepatocyte, transplant, clinical protocol for, 313, 321 (commentary)

Heterozygote, identification, 3-ketothiolase deficiency, 429

HIV: see Human immunodeficiency virus

Hormone, prenatal, antioxidant enzyme gene expression by, fetal rat lung, 171

Human immunodeficiency virus

abnormalities of immune regulation, S71

biologic and immunologic features, S63

cytotoxicity, neonatal and adult leukocytes, 469

symptomatic, intravenous immune globulin prophylaxis, $\mathrm{S} 80$

Human immunodeficiency virus-1

maternofetal transmission, S76

sequence heterogeneity, neonatal blood donor and recipient, 36

Hypercapnia, cerebral vasodilator response to, indomethacin effect, newborn pig, 609

Hyperinsulinemia, glyburide treatment, nondiabetic patients with thalassemia major, 497

Hyperoxia

antioxidant enzyme response to, after prenatal dexamethasone, preterm rat, 177

effect on isolated lung, premature and fullterm lambs, 519

inhibition of lung development by, U74389F treatment effect, newborn rat, 632

pulmonary vasodilation produced by, newborn lamb, 341

Hyperplasia, cellular, small bowel resection, weanling rat, 215

Hyperprostaglandin E syndrome, calcium kinetics in, 92

Hypoglycemia, chronic, cytosolic phosphoenolpyruvate carboxykinase induction by, ovine liver, 493

Hypoinsulinemia, chronic, cytosolic phosphoenolpyruvate carboxykinase induction by, ovine liver, 493

Hypotension, cerebral blood flow during, phenobarbital effect, newborn pig, 598

Hypothalamus, developmental expression of vasopressin in, 152

Hypoxemia, chronic, body fluid compartment volumes in, lamb, 267

Hypoxia

effect on isolated lung, premature and fullterm lambs, 519

environmental, maximum coronary flow rate and, isolated rabbit heart, 527

hemodynamic status after, pancuronium effect, piglet, 365

Hypoxia-ischemia, allopurinol effect, immature rat, 405

Ibuprofen, retinal function deterioration prevented by, after neonatal asphyxia, pig, 336

IgG: see Immunoglobulin $\mathrm{G}$

Iliac artery, in situ morphology, fetal and neonatal rat, 302

Immune globulin

intravenous

autoimmune neuromuscular disease, S95 
prophylaxis, symptomatic HIV infection, $\mathrm{S} 80$

Immunoblot analysis, identification of 3-ketothiolase deficiency, 429

Immunodeficiency

adenosine deaminase deficiency

biochemical abnormalities and molecular genetics, S35

enzyme replacement therapy, $\mathrm{S} 42$

gene therapy for, $S 49$

autoimmune neuromuscular disease, intravenous immune globulin treatment, S95

common variable, T-cell abnormalities, $\mathrm{S} 24$

foreword to supplement, S1

HIV infection

abnormalities of immune regulation, S71

biologic and immunologic features, S63

HIV-1 infection, maternofetal transmission, $\mathrm{S} 76$

human immunoglobulin E response, regulation of, S56

intravenous immune globulin prophylaxis, symptomatic HIV infection, S80

lymphocyte signal transduction pathways, molecular dissection of, $\mathrm{S} 9$

pathogenesis of immune-mediated neuropathies, $\mathrm{S} 90$

severe combined, with selective T-cell cytokine genes, S20

T-cell, transmembrane signaling and, S16

$\mathrm{X}$-linked, prenatal analysis and genetic analysis, S29

yesterday, today, and tomorrow, $\mathrm{S} 2$

Immunoglobulin

binding, epitope mapping of antigen of Dermatophagoides pteronyssinus for, 209

commercial preparations, anti-idiotypic antibodies to poliovirus antibodies in, 475

light chain usage, diversity of, human immune response to $\mathrm{Hib}$ polysaccharide, 307

Immunoglobulin $\mathrm{E}$, response, regulation of, S56

Immunoglobulin $\mathrm{G}$, receptors, cell-surface expression, premature infants, 452

Indomethacin

antenatal administration, hemodynamic effects, fetus and newborn, 615

cerebral vasodilator response to arachidonic acid and hypercapnia after application of, newborn pig, 609

effect on cerebral intracellular oxygenation, preterm infant, 603

effect on polymorphonuclear leukocyte function, preterm infant, 32

myocardial perfusion and performance after, newborn lamb, 295

regulation of ductus arteriosus smooth muscle cell migration by, fetal lamb, 352

Infant

bronchiolitis, respiratory mechanics and occlusion time, 273

endotracheal suctioning, hemodynamic changes during, autonomic nervous system role, 649

Giardia lamblia-associated infection, cellular immune response during, 15

glucocorticoid regulation of Na,K-ATPase, rat lung and kidney, 5

preterm

cell surface expression of $\mathrm{IgG}$ receptors on polymorphonuclear leukocytes and monocytes, 452

cerebral blood flow estimation, near in frared spectroscopy and ${ }^{133}$ xenon, 56

cerebral intracellular oxygenation, indomethacin effect, 603

constant light effect on sleep and wakefulness rhythm, 159

dexamethasone and dietary zinc effect, piglet, 118

effect of medium-chain triglyceride formula, 101

indomethacin effect on polymorphonuclear leukocytes, 32

protein metabolism, intravenous amino acid effect, 106

ventilated, glucose production and oxidation, 583

Infant formula, medium-chain triglyceride, effect on glucose oxidation, preterm infants, 101

Infant nutrition

docosahexaenoic acid status, breast-fed and formula-fed infants, 425

erythrocyte incorporation of ingested iron isotope, breast-fed and formula-fed infants, 573

Inhibin, reduced levels, early pubertal boys with testicular defects, 514

Insulin-like growth factor

binding protein, role of pituitary $\mathrm{GH}$ in regulation of, fetal rat, 144

binding protein-3, characterization chronic renal failure serum, 136

regulation of, role of pituitary $\mathrm{GH}$, fetal rat, 144

Interferon- $\gamma$, combined with macrophagecolony stimulating factor, osteopetrosis ameliorated by, microphthalmic mice, 384

Interleukin-1 $\beta$, plasma levels, neonatal sepsis, 380

Interleukin-6, plasma levels, neonatal sepsis, 380

Interrupter technique, role, measurements of respiratory mechanics, 261, 451 (erratum)

Intestine: see also Jejunum, Small intestine

blood flow regulation, ischemia and reperfusion effects, swine, 400

fatty acid-induced injury, developing piglet, 543

Iron, erythrocyte incorporation of, breast-fed and formula-fed infants, 573

Irradiation, cranial, effect on growth and puberty, acute lymphoblastic leukemia patients, 577

Ischemia, cerebral, vascular effects, piglet, 164

Jaundice, prevention, metalloporphyrin heme oxygenase inhibitors, 195

Jejunum

lactose uptake, newborn piglet, 568

mucosa, immunohistochemical study, Kawasaki disease, 557

permeability, diet effect during diarrhea, suckling rat, 548

Joint contracture, diabetic children, collagen and laminin concentrations and, 501

Kawasaki disease, small intestinal mucosa, immunohistochemical studies, 557

3-Ketothiolase, deficiency, postnatal diagnosis, 429

Kidney

chronic failure, characterization of insulinlike growth factor binding protein-3 in, 136 glucocorticoid regulation of $\mathrm{Na}^{+}, \mathrm{K}$ ATPase mRNA, developing rat, 5

recovery of adenosine triphosphate, postischemic thyroxin infusion effect, rat, 595

Labor, effect on polymorphonuclear leukocyte function, healthy neonate, 466

Lactate, role in lipogenesis, fetal rat brain, 66

Lactic acid, bacteria, macromolecular absorption and, suckling rat, 548

Lactic acidosis, adenine nucleotide translocator deficiency in patient with, 412

Lactose, uptake, newborn colon and jejunum, piglet, 568

Laminin, circulating metabolites, insulin-dependent diabetics with joint and skin manifestations, 501

Lawson Wilkins Pediatric Endocrine Society, joint meeting with European Society for Paediatric Endocrinology, abstracts, 5S1

Leucine, whole body oxidation, noninvasive estimation, maple syrup urine disease, 445

Leukemia, acute lymphoblastic, treatment effects on growth and puberty, 577

Leukocyte

cytotoxicity of HIV-infected cells by, neonates and adults, 469

polymorphonuclear

cell-surface expression of $\mathrm{IgG}$ receptors, premature infants, 452

chemotaxis, leukotriene $\mathrm{B}_{4}$ and, 242

maternal labor and delivery mode effect, 466

Leukotriene $\mathrm{B}_{4}$, polymorphonuclear leukocyte chemotaxis to, neonate, 242

Light-dark cycle, role, sleep and wakefulness, preterm infants, 159

Light exposure, effect on TPN solutions, rat, 487

Lipid

mucosal injury and, developing intestine, piglet, 543

peroxidation, role, metalloporphyrin-mediated phototoxic reactions, neonatal rat, 87

plasma concentrations, changes, prepubertal psoriatic children, 506

Lipogenesis, lactate role, fetal rat brain, 66

Liver

effect of chronic fetal hypoglycemia and hypoinsulinemia, fetal sheep, 493

hepatic gene transfer, clinical protocol for, 313, 321 (commentary)

toxicity, carbon tetrachloride, developmental resiliency to, neonatal rat, 225

TPN-associated alterations, light exposure role, rat, 487

Lung

antioxidant enzyme gene expression, prenatal hormone effect, fetal rat, 171

distension, spontaneous fetal breathing and, hemodynamic effect, sheep, 639

fluid balance, hypoxic-hyperoxic effects, premature and full-term lambs, 519

glucocorticoid regulation of $\mathrm{Na}^{+}, \mathrm{K}^{+}-\mathrm{AT}$ Pase mRNA, developing rat, 5

hyperoxic injury

intratracheal antioxidant treatment, rat, 332

U74389 treatment effect, rat, 632

hypertension: see Pulmonary hypertension

maturation, after maternal betamethasone, preterm rabbit, 256

mechanics, measurements, volume history and, rabbit, 261, 451 (erratum) 
nuclear retinoic acid receptors, gestational age and retinol deficiency effects, fetal rat, 251

oxygen-induced vasodilation, adenosine triphosphate and adenosine role, fetal lamb, 533

vasodilation, role of endothelium-derived nitric oxide during, newborn lamb, 341

Lung surfactant, antenatal cocaine exposure effect, late gestation rat, 327

Lymphoblast, peroxisome analysis, Zellweger syndrome, 441

Lymphocyte

actions of RNA/mononucleotides on, 458

peripheral blood subsets, during Giardia lamblia-associated diarrhea, 15

signal transduction pathways, molecular dissection of, $\mathrm{S} 9$

Macrophage-colony stimulating factor, combined with interferon- $\gamma$, osteopetrosis ameliorated by, microphthalmic mice, 384

Maple syrup urine disease, whole body leucine oxidation in, estimation methods, 445

Meningitis

autoregulatory impairment, Haemophilus influenzae type b effect on pial vessels, rat, 48

bacterial, amino acid role in CNS injury, rabbit, 510

Messenger ribonucleic acid, glucocorticoid effect on regulation of, infant rat heart, 1

Metabolism, 3-ketothiolase deficiency, heterozygote diagnosis, 429

Methotrexate, effect on growth and puberty, acute lymphoblastic leukemia patients, 577

Mitochondria, disorder, neonatal cardiopulmonary arrest and, 433

Monocyte, cell-surface expression of IgG receptors, premature infants, 452

Mononuclear cells, cell surface phenotypes, small intestinal mucosa, Kawasaki disease, 557

Motor neuropathy, E1 pyruvate dehydrogenase deficiency in child with, 284

Mucin, colonic, age and diet effect, pig, 564

Mucosal immune response, after upper respiratory tract infection, SLDS infant, 554

Multinucleated giant cell, formation, umbilical cord and adult mononuclear phagocytes, 19

Multiple acyl-CoA dehydrogenation disorder, riboflavin effect, 129

Myopathy, mitochondrial, adenine nucleotide translocator deficiency in patient with, 412

$\mathrm{Na}^{+} / \mathrm{H}^{+}$exchanger activity, bowel resection effect, weanling rat, 215

Neonate

asphyxia, ibuprofen effect on retinal function deterioration after, pig, 336

carbon tetrachloride hepatotoxicity, chlordecone role, rat, 225

cardiopulmonary arrest, mitochondrial disorder associated with, 433

cerebral vasodilator response to arachidonic acid and hypercapnia, newborn pig, 609

cerebral venous saturation, estimation by near infrared spectroscopy, 52

circulatory effects of epinephrine infusion, piglet, 190 direct ventricular interaction, lamb, 359

dopamine receptor blockade and stimulation, systemic and pulmonary vascular effects, lamb, 181

energy substrate utilization, comparison of TPN regimens, 112

extracorporeal membrane oxygenation, cerebral autoregulation during, lamb, 289

group B $\beta$-hemolytic streptococcus, straindependent differences in hemodynamic response, lamb, 373

hemodynamic effects of antenatal indomethacin, randomized study, 615

hemorrhagic hypotension, phenobarbital effect on cerebral blood flow during, piglet, 598

hypoxia, hemodynamic effects of pancuronium after, piglet, 365

immature, glucose production rate, 97

indomethacin administration, myocardial perfusion after, lamb, 295

jaundice, screening of metalloporphyrin heme oxygenase inhibitors, 195

lactose flux mechanisms, colon and jejunum, piglet, 568

lung development, hyperoxic inhibition, U74389F protection against, rat, 632

neutrophil, diminished actin polymerization by, 27

neutrophil chemotaxis to leukotriene $B_{4}$, 242

normoxia, hemodynamic effects of pancuronium after, piglet, 365

peripheral circulation, monitoring by plethysmo time interval, 653

phototoxic reactions, lipid peroxidation role, rat, 87

polymorphonuclear leukocyte function, maternal labor and delivery mode effect, 466

pulmonary vasodilation, role of endothelium-derived nitric acid during, newborn lamb, 341

retinal circulation, adenosine effect, pig, 620

rotavirus-associated biliary obstruction, mice, 394

sepsis, tumor necrosis factor- $\alpha$, interleukin- $1 \beta$, and interleukin- 6 plasma levels, 380

soluble receptors for tumor necrosis factor$\alpha$ in, 82

ventilated, glucose metabolism in, 583

zinc status, prenatal maternal zinc deficiency and, monkey, 233

Neural crest

ablation, $v s$ sham surgical effects, chick embryo, 628

proteins associated with, early chick embryo, 43

Neuromuscular disease, autoimmune, intravenous immune globulin treatment S95

Neuropathy, immune-mediated, pathogenesis of, $\mathbf{S} 90$

Neutrophil, actin polymerization by, neonate, 27

Nitric oxide, endothelium-derived, role during pulmonary vasodilation, newborn lamb, 341

Normoxia, hemodynamic status after, pancuronium effect, piglet, 365

Nutrition, maternal, zinc deficiency effect on pregnancy outcome, monkey, 233

Nylidrin hydrochloride, antenatal administration, hemodynamic effects, fetus and newborn, 615
Occlusion time, role, respiratory mechanics, infants with bronchiolitis, 273

Ornithine carbamoyl transferase, deficiency, magnetic resonance spectroscopy role, 77

Osteopetrosis, effect of macrophage-colony stimulating factor and interferon- $\gamma$ administration, microphthalmic mice, 384

Oxygen

arteriovenous content, ischemia and reperfusion effect, postnatal intestine, swine, 400

pulmonary toxicity, mitigation by intratracheal antioxidants, rat, 332

transport regulation, univariate genetic analysis, twin study, 645

Oxygenation, extracorporeal membrane, cerebral autoregulation during, newborn lamb, 289

Oxygen saturation, cerebral venous, estimation, near infrared spectroscopy, neonate, 52

Pancuronium, effect on hemodynamic status, after normoxia or hypoxia, piglet, 365

Pantothenate, role, reversal of chronic 2- $n$ propyl-4-pentenoic acid hepatotoxicity, mice, 72

Parenteral nutrition

bisulfite effects, human vascular tissue, 347 total

energy metabolism in neonate receiving, 112

light exposure of solutions for, rat, 487

Permeability barrier, ontogenesis preservation, intrauterine growth-retarded $\mathrm{fe}$ tal rat, 418

Peroxisome, disorder, lymphoblast role, Zellweger syndrome, 441

Phenobarbital, effect on cerebral blood flow, during hemorrhagic hypotension, newborn piglet, 598

Phosphoenolpyruvate carboxykinase, induction by chronic fetal hypoglycemia and hypoinsulinemia, ovine liver, 493

Phototoxicity, metalloporphyrin-mediated, lipid peroxidation role, neonatal rat, 87

Pial vessel, autoregulation, Haemophilus influenzae type b impairment of, rat, 48

Pituitary, regulation of late-gestation fetal growth by, rat, 144

Plethysmo time interval, clinical value, evaluation of neonatal peripheral circulation, 653

Poliovirus, antibodies, anti-idiotypic antibodies to, commercial Ig, human sera, and milk, 475

Polyethylene glycol, -conjugated antioxidant enzymes, intratracheal instillation, hyperoxic lung injury, rat, 332

Polymorphonuclear leukocyte: see Leukocyte

Pregnancy

cocaine abuse during, effect on surfactant and antioxidant enzyme maturation during, rat, 327

outcome, after maternal zinc deficiency, monkey, 233

soluble receptors for tumor necrosis factor$\alpha$ during, 82

2-n-Propyl-4-pentenoic acid, chronic hepatotoxicity, reversibility of, mice, 72

Prostaglandin, production, parenteral bisulProtein 
cardiac neural crest-associated, early chick embryo, 43

metabolism, intravenous amino acid effect, preterm infants, 106

Psoriasis, plasma lipid and lipoprotein alterations, prepubertal patients with, 506

Puberty, after acute lymphoblastic leukemia treatment, cranial irradiation effect, 577

Pulmonary hypertension, mechanically increased right ventricular afterload effect, neonatal lamb, 359

Pulse oximeter, monitoring peripheral circulation by, neonate, 653

Respiratory tract infection, mucosal immune

Retina response after, SIDS infant, 554

microcirculatory responses to adenosine, newborn pig, 620

postasphyxial injury, ibuprofen effect newborn pig, 336

Riboflavin, multiple acyl-coenzyme A dehydrogenation disorder responsive to, 129

Ribonucleic acid/nucleotides

as immodulating nutrients, 458

$\mathrm{IgM}$ and $\mathrm{IgG}$ production enhanced by, 458

Rotavirus

enteritis, diet effect on jejunal permeability, suckling rat, 548

group A, extrahepatic biliary obstruction produced by, newborn mice, 394

Sepsis

group B streptococcal, diaphragmatic function during, piglet, 10

neonatal, cytokine plasma levels during, 380

Serine, transplacental transport, comparison with leucine, sheep, 590

SIDS: see Sudden infant death syndrome
Skin manifestations, diabetic children, collagen and laminin concentrations and, 501

Sleep, light-dark cycle effect, preterm infants, 159

Small intestine, resection, $\mathrm{Na}^{+} / \mathrm{H}^{+}$exchanger activity and, weanling rat, 215

Smoking, passive, oxygen transport regulation and, genetic analysis, 645

Sodium pump, mRNA, glucocorticoid effect, infant rat heart, 1

Spectroscopy

magnetic resonance, role, ornithine carbamoyl transferase deficiency, 77

near infrared, cerebral venous saturation estimated by, neonate, 52

Streptococcus

group B, sepsis, diaphragmatic function during, piglet, 10

group $\mathrm{B} \beta$-hemolytic, strain-dependent differences in hemodynamic response, neonatal lamb, 373

Sudden infant death syndrome, mucosal immune response and, 554

Superoxide scavenger, pretreatment, effect on ischemia-induced alteration of cerebral vasodilation, piglet, 164

Testosterone, role, gonadotropin secretion control, peripubertal boys, 514

Tetrahydrobiopterin, catalytic activity, in dihydropteridine reductase deficiency, 125

Thalassemia major, glyburide treatment of hyperinsulinemia in nondiabetic patients with, 497

The American Pediatric Society and The Society for Pediatric Research, abstracts of annual meeting, 3A, 657 (erratum)

Thiamine, supplementation, maple syrup urine disease, 445
Thyroxin, postischemic infusion, effect on recovery of renal ATP, rat, 595

TPN: see Parenteral nutrition, total

Transforming growth factor- $\alpha$, expression, fetal gastrointestinal tract, 481

Transforming growth factor- $\beta 1$, clonal growth regulation by, human fetal chondrocytes, 390

Tricuspid valve, antenatal indomethacin effect, fetus and newborn, 615

Triglyceride, medium-chain, decreased glucose oxidation in preterm infants receiving, 101

Tumor necrosis factor- $\alpha$

plasma levels, neonatal sepsis, 380

soluble receptors, pregnant women serum and neonatal urine, 82

U74389F, protective effect, hyperoxic inhibition of lung development, newborn rat, 632

Vasopressin, developmental expression, human hypothalamus, 152

Visual-evoked potential, erythrocyte docosahexaenoic acid and, healthy term infants, 425

Vitamin A, role, regulation of retinoic acid receptors, fetal rat lung, 251

${ }^{133}$ Xenon, cerebral blood flow estimated by, comparison, near infrared spectroscopy, 56

Zellweger syndrome, characterization of perZinc oxisomes in lymphoblasts, 441

dietary, zinc homeostasis and, piglet, 118

maternal deficiency, pregnancy outcome and infant zinc status, monkey, 233

metabolism, effect of dexamethasone or excess dietary zinc, piglet, 118 